\title{
Numerical study of surface tension effects on bubble detachment in a submerged needle
}

\author{
J. Eshraghi, E. Kosari, P. Hadikhani, A. Amini, \\ M. Ashjaee \& P. Hanafizadeh \\ Centre of Excellence in Design and Optimization of Energy Systems, \\ School of Mechanical Engineering, College of Engineering, \\ University of Tehran, Iran
}

\begin{abstract}
This paper analyses adiabatic gas bubble formation, growth, and detachment which are produced from a submerged needle in water with the aim of elucidating the impact of surface tension on bubble properties. A simulation model is utilized to enable examination of the problem for the mentioned parameter. The numerical results are compared with experimental observations on the bubble for validation purposes. The simulation results, including changes in bubble properties during the process, are recorded and a comprehensive research for different surface tensions is applied to understand the effect of this parameter on the bubble. It is found that higher surface tension between gas and water results in larger bubbles right before the departure occurs.

Keywords: bubble formation and growth, experimental observations, simulation model, surface tension.
\end{abstract}

\section{Introduction}

The bubble formation within submerged needles or orifices occurs in a large number of technical applications, including reactors, water treatment instruments, and a variety of chemical plants, such as perforated plate columns. This phenomenon has also some resemblance to bubble departure in boiling, which is a major aspect of industrial operations in the power generation industry. Bubble columns are one of the most appropriate apparatuses to simulate bubble formation under industrial conditions, which have been utilized in numerous multi-phase researches, due to their simple structure and high performance. 
Owing to widespread use of this issue, there are many experimental and analytical studies on bubble formation and growth. For the case of CFD simulation methods for multi-phase flows, Eulerian-Eulerian (E-E), EulerianLagrangian (E-L) and direct numerical simulation (DNS) methods have been employed (Delnoij et al. [1], Krishna and van Baten [2], Yang et al. [3], Das et al. [4], Di Bari and Robinson [5]). One of the most important parameters in formation and dynamics of bubbles is surface tension, especially in boiling process (Weinberg [6], Loubière and Hébrard [7], Zhang et al. [8]).

The objective of the present study is to examine the shape and volume of detaching bubbles in the regime of moderate growth rate. As mentioned, surface tension has been studied as the main effective parameter in extensive articles. In this study, positive influences of these issues on the bubble detachment volume are discussed, and the changes of bubble diameter, and bubble formation frequency are demonstrated. Finally, a comprehensive investigation on surface tension effects in bubble formation is introduced.

\section{Methodology}

In this section the proposed numerical model and experimental setup are introduced. To use the proposed model for analysing complicated cases with acceptable confidence, it is necessary to study the compatibility of numerical results with those obtained from running the experiment. To do this, a specific and simple case of air being injected into water is chosen as the bench mark problem to be studied experimentally and numerically. The results obtained from the two methods are then compared to judge the accuracy and precision of the numerical model.

\subsection{Numerical model}

The geometry under examination consists of a cube with a square cross section at the bottom of which a needle is installed. The cube is filled with water that forms a liquid column. The gas phase is injected into the liquid medium from the needle hole. Once the meshed geometry is ready, the following equations and the corresponding solution method could be applied to solve the problem numerically.

In this study, the fluid can be assumed incompressible because of satisfying the following conditions: (1) the gas velocity is low and the Mach number (the ratio of the fluid velocity to ambient sound velocity) is far less than 0.2, i.e. the fluid velocity is far less than the sound velocity; (2) the needed time for an obvious change of gas velocity can be considered as the time step length $(0.0005 \mathrm{~s})$ and in the process of bubble generation, the characteristic length of flow domain is of the same order of magnitude with the needle size $(1.6 \mathrm{~mm})$ (VanSintAnnaland et al. [9], Rabha and Buwa [10]). In fact, the volume and pressure variation of gas chamber in the bubble formation are relatively small. Hence the gas is treated as an incompressible fluid. Continuity equation for an incompressible Newtonian liquid is as follows: 


$$
\nabla \cdot \vec{u}=0 \quad \rightarrow \quad \frac{\partial u}{\partial x}+\frac{\partial v}{\partial y}+\frac{\partial w}{\partial z}=0
$$

Momentum equation for an incompressible Newtonian liquid can be written as follows:

$$
\partial(\rho \vec{u}) / \partial t+\nabla \cdot(\rho \vec{u} \vec{u})=-\nabla p+\nabla \cdot\left\{\mu\left[\nabla \vec{u}+(\nabla \vec{u})^{T}\right]\right\}+F_{s}+\rho \vec{g}
$$

Since density is assumed to be constant, it can be easily factorized from the above equation.

In VOF model, the method of tracing inter-phase boundary is achieved by solving volume fraction continuity equation of one phase or several phases. As for the $j$ phase, volume fraction equation is:

$$
\partial F_{j} / \partial t+\overrightarrow{u_{j}} \cdot \nabla F_{j}=0
$$

Eqn (3) does not solve for the primary phase; primary phase volume fraction calculation is based on the following constraints:

$$
\sum_{j=1}^{2} F_{j}=0
$$

The calculating method of density and viscosity of the mixed fluid in a computational cell is as follows:

$$
\begin{aligned}
& \rho(\vec{x}, t)=F(\vec{x}, t) \rho_{l}+[1-F(\vec{x}, t)] \rho_{g} \\
& \mu(\vec{x}, t)=F(\vec{x}, t) \mu_{l}+[1-F(\vec{x}, t)] \mu_{g}
\end{aligned}
$$

The surface tension force term in eqn (2) is considered by applying the continuum surface force (CSF) model proposed by Brackbill et al. [11]. In this model, the surface tension force is considered to be constant along the surface, and only the forces normal to the interface are considered. The surface tension force at the bubble-liquid interface is expressed as a volume force in the momentum equation, using the divergence theorem. This volume force for gas and liquid in the mixture is given by:

where

$$
F_{s}=\sigma\left[2 \rho \kappa \nabla F_{l} /\left(\rho_{g}+\rho_{l}\right)\right]
$$

$$
\kappa=\nabla . \hat{n}, \hat{n}=\frac{n}{|n|}, n=\nabla F_{j}
$$

Uncoupling arithmetic method was used to separate and solve the equations represented by the model; pressure-velocity coupling was the pressure-implicit with splitting of operators (PISO) method, pressure discretization method was pressure staggering option (PRESTO), and momentum discretization method was QUICK mode.

\subsection{Experimental approach}

As the goal of this study is to analyse the bubble growth and find the bubble detachment criteria, the changes in bubble size have to be observed. The implemented experimental setup guarantees the obtaining of this information by using a high-speed camera to define the bubble size and shape. In this section, in order to validate the numerical analysis presented in previous sections, one case is examined experimentally. In the following, experimental set-up is described and results related to applying air as the injected gas are demonstrated and compared with those obtained from the numerical approach. 


\subsubsection{The experimental set-up}

The experimental set-up consists of a transparent column fitted with a bubble injection system and two $800 \mathrm{~W}$ projectors installed to produce sufficient light to obtain bubble growth images. The experimental set-up is depicted in Figure 1. The $100 \times 100 \times 300 \mathrm{~mm}^{3}$ column has a square cross-section. The injection system is composed of a very thin needle (Needle Gauge 14) connected to an automatically controlled syringe (JMS P500) and placed $15 \mathrm{~mm}$ upper from the bottom of the column. The syringe is filled with air and the flow rate is set to $300 \mathrm{ml} / \mathrm{hr}$ to produce only a single bubble at time.

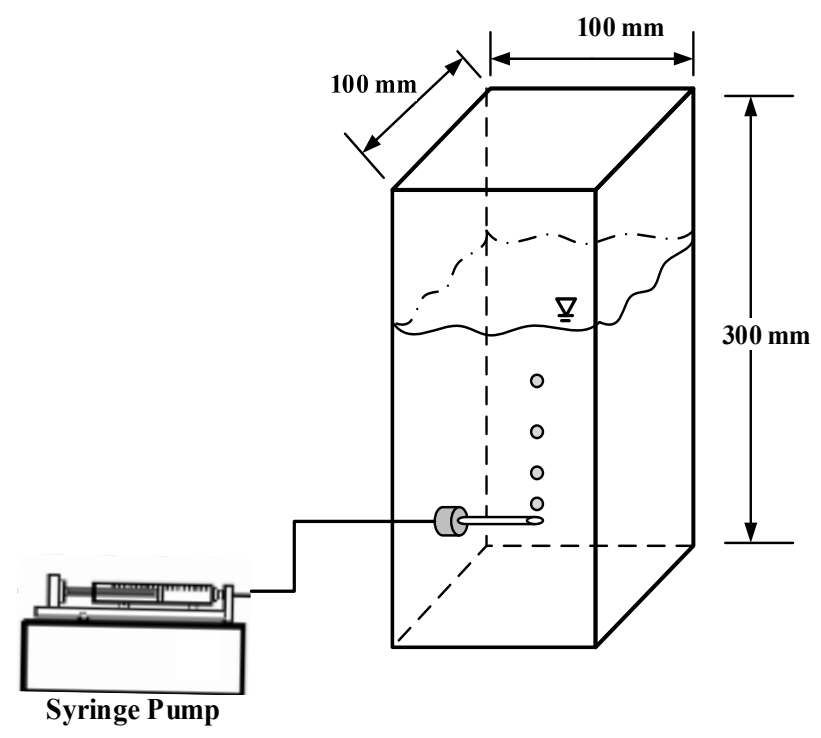

Figure 1: Schematic view of experimental setup.

All images collected from the experiments are analysed with an image processing code in MATLAB in order to obtain required data for the study.

\subsubsection{Validation of the numerical model}

The evolution of the bubbles shape during the bubble growth and detachment process in purified water is shown in Figure 2. It can be clearly seen that in the studied case of bubble formation in water, bubbles expand spherically at the beginning then evolve symmetrically from a spherical shape to an inverse teardrop shape with the vertical axis passing through the needle centre. The first shape is due to the strength of the surface tension at the beginning of the bubble formation. Subsequently, the influence of bubble buoyancy gradually increases with the bubble growth as a result of more injected gas which tends to move upward. Thus, the interface moves upward and the bubble elongates to an obvious teardrop shape. Changes in viscosity and other physical properties affect the bubble shape, but not the general formation mechanism. 


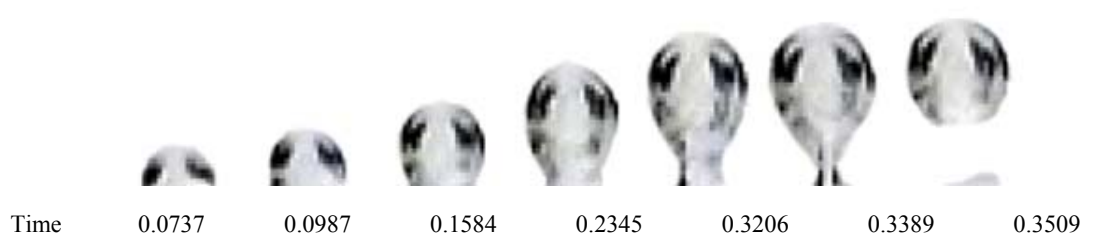

(a)

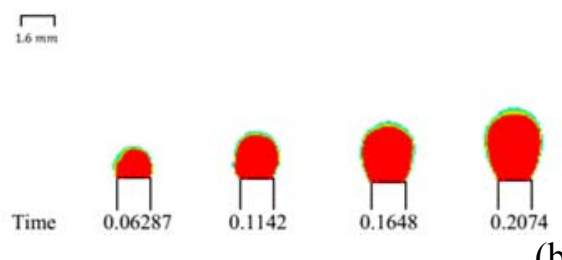

(b)
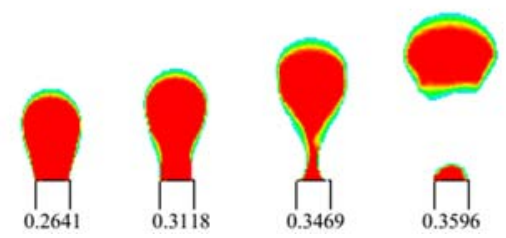

Figure 2: Bubble growth process at the needle, until the floating begins. The time is shown in seconds. (a) Experimental results; (b) Numerical results.

Figure 3 shows the changes in bubble diameter. Also, Table 1 compares the results obtained from the two approaches, and demonstrates the bubble critical volume at the detachment. The results gained from the numerical approach show great compatibility with those obtained from the experiment. This is a good proof for the validity of the numerical solution and analysis.

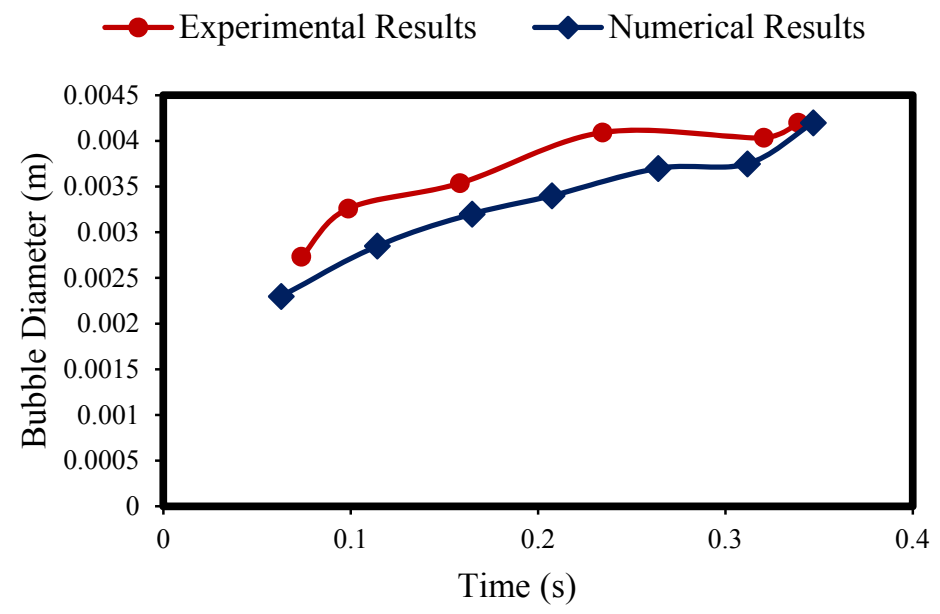

Figure 3: Changes in diameter of air bubbles: a comparison between numerical and experimental results. 
Table 1: A comparison between air bubble critical volumes at the detachment obtained from the numerical and experimental approaches.

\begin{tabular}{lc}
\hline \hline Approach & $\begin{array}{c}\text { Bubble volume at } \\
\text { departure }\left(\mathrm{m}^{3}\right)\end{array}$ \\
\hline Experimental & $4.759 \times 10^{-8}$ \\
Numerical & $6.160 \times 10^{-8}$ \\
\hline \hline
\end{tabular}

\section{Results and discussion}

In the previous section, in order to test the accuracy and robustness of the numerical method, numerical prediction of bubbles were validated against experimental observation of the specific case of air injected into water. Based on this validation, the numerical method is further applied to investigate the effects of the surface tension on the bubble size. In this section, investigation of effects of surface tension on the bubble formation process has been planned, the model has been applied to three different mixtures that represent different values of surface tension. The following contains the associated results and discussions.

Results are presented for three different cases of study with different properties, in order to discover the importance and effect of surface tension. The properties of the injected gases are displayed in Table 2.

Table 2: Injected gas properties.

\begin{tabular}{lccc}
\hline \hline Test case & Density $\left(\mathrm{kg} / \mathrm{m}^{3}\right)$ & Viscosity $(\mathrm{kg} / \mathrm{m} . \mathrm{s})$ & $\begin{array}{c}\text { Surface tension } \\
(\mathrm{N} / \mathrm{m})\end{array}$ \\
\hline Case 1: Air & 1.225 & 0.000018 & 0.073 \\
Case 2: Nitrogen & 1.251 & 0.000018 & 0.070 \\
Case 3: Helium & 0.1786 & 0.000019 & 0.071 \\
\hline \hline
\end{tabular}

Figure 4 shows the sequence of bubble formation in the vicinity of the needle in water for different injected gases. The main periods of bubble evolution, i.e. bubble nucleation at the initiation and bubble growth, include several meaningful stages, such as under critical growth, and critical growth and necking, which extends until the bubble floating step starts. Although the mechanism of bubble evolution is similar regardless to wetting conditions, differences in bubble shape, volume, and growth kinetics during each period are revealed. Figure 4(a) illustrates thoroughly the stages of bubble evolution.

Considering numerical model proposed in previous sections, in the following, the results are presented in the case of changes in bubble diameter, volume. In order to achieve a reliable and appropriate conclusion, the effects of surface tension on bubble growth, and changes in bubble diameter during growth are displayed in Figure 5. 


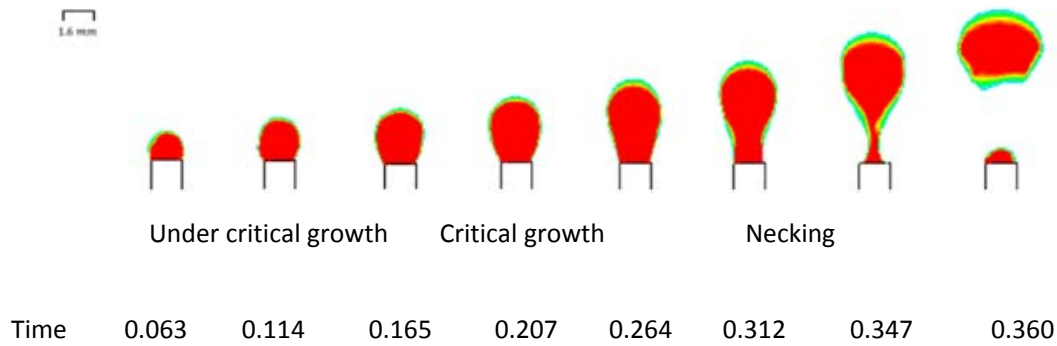

(a)

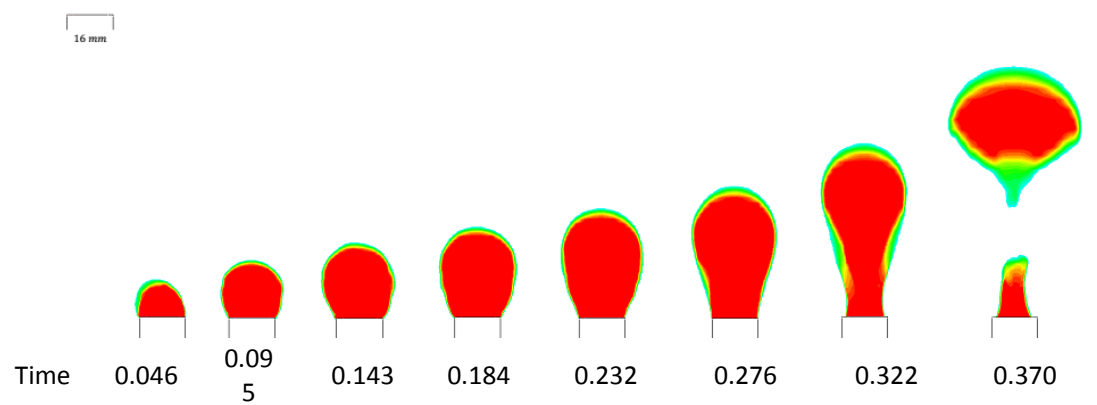

(b)

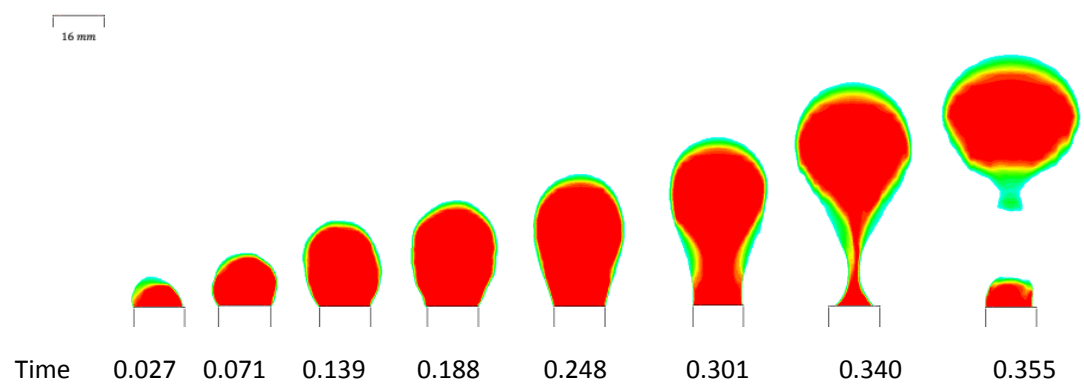

(c)

Figure 4: Bubble growth process at the orifice, until the floating begins. The time is shown in seconds: (a) First case: $\boldsymbol{\sigma}=\mathbf{0 . 0 7 3} \mathbf{N} / \boldsymbol{m}$; (b) Second case: $\sigma=0.070 \mathrm{~N} / \mathrm{m}$; (c) Third case: $0.071 \mathrm{~N} / \mathrm{m}$ (theoretical results). 
$\smile$ Surface Tension $=0.073 \mathrm{~N} / \mathrm{m} \longrightarrow$ Surface Tension $=0.070 \mathrm{~N} / \mathrm{m}$

$\longrightarrow$ Surface Tension $=0.071 \mathrm{~N} / \mathrm{m}$

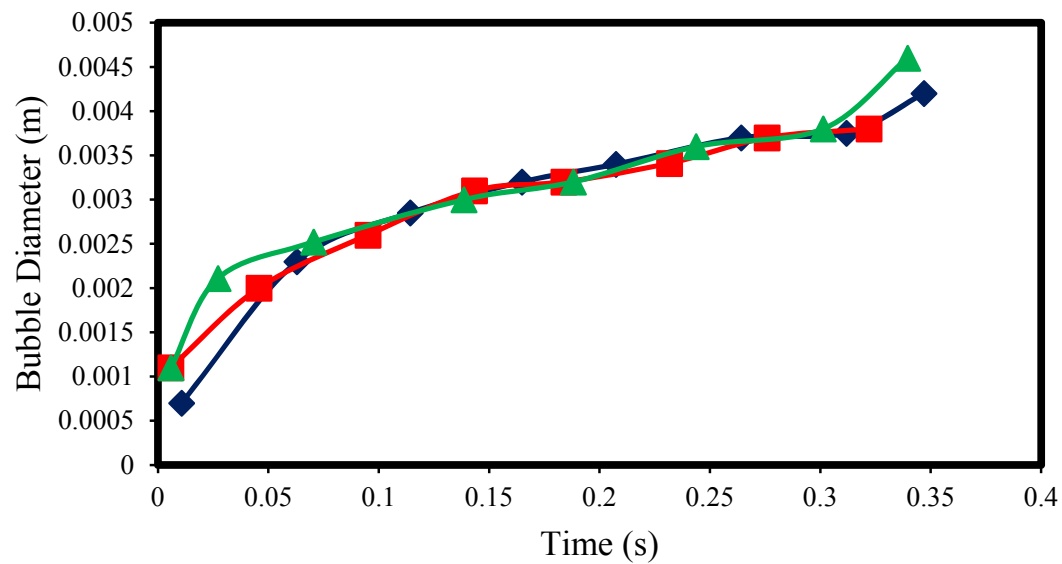

Figure 5: Changes in bubble diameter for different surface tensions.

Thus, by assuming spherical shape of bubbles, the changes in bubble volume is shown in Figure 6.

$\prec$ Surface Tension $=0.073 \mathrm{~N} / \mathrm{m}$

- Surface Tension $=0.070 \mathrm{~N} / \mathrm{m}$

$\multimap$ Surface Tension $=0.071 \mathrm{~N} / \mathrm{m}$

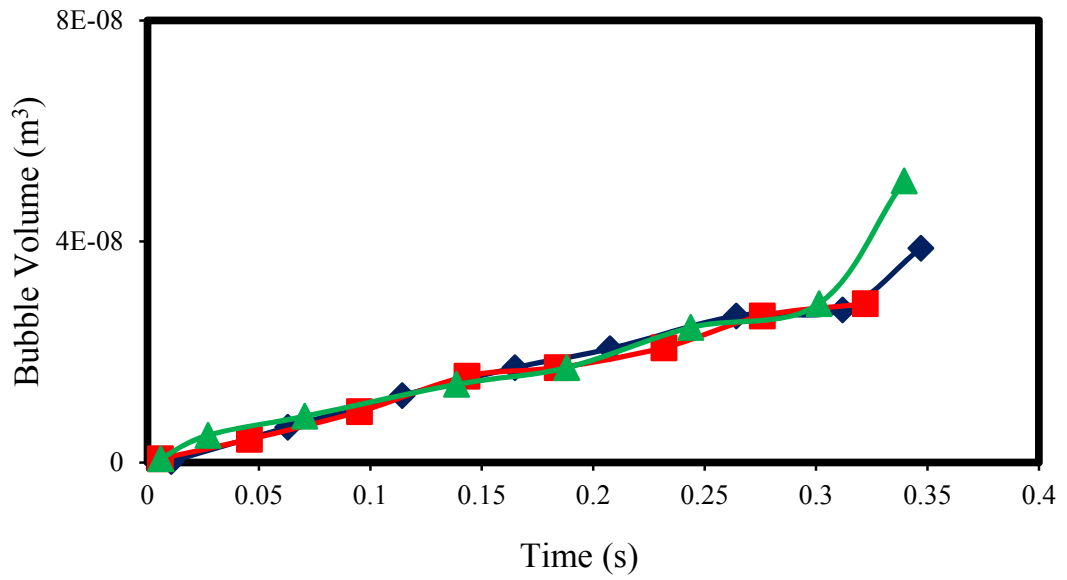

Figure 6: Changes in bubble volume for different surface tensions. 
As it is obvious from Figure 6, the volume of the bubble is growing uniformly, because the velocity of the bubble is less than the mainstream velocity. At the cut-off stage, the departure condition and bubble generation frequency of each case (with different surface tensions) are compared in Table 3.

Table 3: Comparison between bubble critical volume and bubble generation frequency for different cases.

\begin{tabular}{lcc}
\hline \hline Test case & $\begin{array}{c}\text { Bubble volume at } \\
\text { departure }\left(\mathrm{m}^{3}\right)\end{array}$ & $\begin{array}{c}\text { Bubble generation } \\
\text { frequency }(1 / \mathrm{s})\end{array}$ \\
\hline Case 1: $\sigma=0.073 \mathrm{~N} / \mathrm{m}$ & $6.160 \times 10^{-8}$ & 3.0166 \\
Case 2: $\sigma=0.070 \mathrm{~N} / \mathrm{m}$ & $4.460 \times 10^{-8}$ & 3.0677 \\
Case 3: $\sigma=0.071 \mathrm{~N} / \mathrm{m}$ & $6.545 \times 10^{-8}$ & 2.9771 \\
\hline \hline
\end{tabular}

By comparing the bubble departure volume for different gases, it is understood that it should also be considered that gas density and viscosity have influences on the bubble volume. In the third test case, since the gas has a much small density than the liquid, the buoyancy force that act on the bubble is greater than other test cases, so the bubble cuts off earlier and in smaller diameter, in comparison to other test cases. The first and second test cases have almost the same densities, so the surface tension effect can be studied more specifically between these cases. In order to observe the surface tension effects on bubble formation specifically, in the next step, all gas properties keep constant except the surface tension between the gas and the liquid. In this case, numerical model presents the following results, as it is shown in Table 4.

Table 4: Surface tension effects on gas bubble departure.

\begin{tabular}{lccc}
\hline \hline Test case & $\begin{array}{c}\text { Bubble diameter } \\
\text { at departure }(\mathrm{m})\end{array}$ & $\begin{array}{c}\text { Bubble volume at } \\
\text { departure }\left(\mathrm{m}^{3}\right)\end{array}$ & $\begin{array}{c}\text { Bubble generation } \\
\text { frequency }(1 / \mathrm{s})\end{array}$ \\
\hline Case 1: $\sigma=0.04 \mathrm{~N} / \mathrm{m}$ & 0.0046 & $5.096 \times 10^{-8}$ & 5.219 \\
Case 2: $\sigma=0.073 \mathrm{~N} / \mathrm{m}$ & 0.0049 & $6.160 \times 10^{-8}$ & 3.043 \\
Case 3: $\sigma=0.09 \mathrm{~N} / \mathrm{m}$ & 0.0058 & $1.022 \times 10^{-7}$ & 2.603 \\
Case 4: $\sigma=0.1 \mathrm{~N} / \mathrm{m}$ & 0.0061 & $1.189 \times 10^{-7}$ & 2.332 \\
\hline \hline
\end{tabular}

It can be concluded from Table 4 that increased surface tension - specifically surface tension, not other gas properties - results in greater bubbles right before the departure occurs. In addition, as expected, since constant flow condition has been applied to the model, the bubble volume changes affect frequency.

\section{Conclusion}

In this study, a numerical investigation of effects of surface tension on the gas bubble detachment volume are presented and discussed. Also, changes in bubble diameter and bubble formation frequency are all demonstrated as a function of the surface tension. The gas phase is injected into the water column through a 
submerged needle under constant flow condition in order to produce bubbles. The exiting gas from the needle tends to create a bubble with spherical configuration, as a result of the surface tension induced. Based on the presented results, it can be inferred that the volume of generated bubbles increases as the surface tension increases. In addition, it is discovered that on the frequency of bubble generation, surface tension has a less crucial effect than density has. Larger differences in density between water and the injected gas result in larger buoyancy forces which accelerate the bubble detachment, and subsequently the bubble generation frequency increases.

\section{References}

[1] Delnoij, E., Kuipers, J.A.M., van Swaaij, W.P.M., Computational fluid dynamics applied to gas-liquid contactors. Chemical Engineering Science, 52, 3623-3638, 1997.

[2] Krishna, R., van Baten, J.M., Scaling up bubble column reactors with the aid of CFD. Chemical Engineering Research and Design, 79, 283-309, 2001.

[3] Yang, G.Q., Du, B., Fan, L.S., Bubble formation and dynamics in gasliquid-solid fluidization - a review. Chemical Engineering Science, 62, 2-27, 2007.

[4] Das, A.K., Das, P.K., Saha, P., Formation of bubbles at submerged orifices - Experimental investigation and theoretical prediction. Experimental Thermal and Fluid Science, 35, 618-627, 2011.

[5] Di Bari, S., Robinson, A.J., Experimental study of gas injected bubble growth from submerged orifices. Experimental Thermal and Fluid Science, 44, 124-137, 2013.

[6] Weinberg, M.C., Surface tension effects in gas bubble dissolution and growth. Chemical Engineering Science, 36, 137-141, 1981.

[7] Loubière, K., Hébrard, G., Influence of liquid surface tension (surfactants) on bubble formation at rigid and flexible orifices. Chemical Engineering and Processing: Process Intensification, 43, 1361-1369, 2004.

[8] Zhang X.B., Xiang, S., Cao, X., Qiu L.M., Effects of surface tension on bubble growth in an extensive uniformly superheated liquid. Chinese Science Bulletin, 56, 3191-3198, 2011.

[9] VanSintAnnaland, M., Deen, N.G., Kuipers, J.A.M., Numerical simulation of gas bubbles behaviour using a three-dimensional volume of fluid method. Chemical Engineering Science, 60, 2999-3011, 2005.

[10] Rabha, Swapna S., Buwa, Vivek V., Volume-of-fluid (VOF) simulations of rise of single/multiple bubbles in sheared liquids. Chemical Engineering Science, 65, 527-537, 2010.

[11] Brackbill, J.U., Kothe, D.B., Zemach, C., A continuum method for modelling surface tension. Journal of Computational Physics, 100, 335-354, 1992. 